\title{
Cost of Hospitalizations due to Exacerbation in Patients with Non-Cystic Fibrosis Bronchiectasis
}

\author{
David de la Rosa Carrillo ${ }^{a}$ Annie Navarro Rolon ${ }^{b}$ Rosa Maria Girón Moreno ${ }^{c}$ \\ Beatriz Montull Veiga $^{d}$ Casilda Olveira Fuster ${ }^{e}$ Alicia Padilla Galo $^{f}$ \\ Concepción Prados Sánchez ${ }^{g}$ Esther Quintana Gallego ${ }^{\text {h }}$ Oriol Sibila Vidal ${ }^{i}$ \\ Nuria Celorrio Jiménez ${ }^{j}$ Antonio Ruiz Peña ${ }^{k}$ Antoni Torres Martíl Maria Jesús Avilés Inglés ${ }^{m}$ \\ Marina Blanco Aparicio ${ }^{n}$ Marta García-Clemente ${ }^{\circ}$ Rafael Golpe Gómez ${ }^{p}$ \\ Ainhoa Gómez Bonillaq Cristina Gómez González ${ }^{r}$ Maria Victoria Leal Arranz ${ }^{s}$ \\ Patricia Mínguez Clemente ${ }^{t}$ Belén López Muñiz ${ }^{u} \quad$ Luis Máiz Carro $^{v} \quad$ Ana Pando Sandoval ${ }^{\circ}$ \\ Juan Luis Rodríguez Hermosaw Ane Uranga Echeverria ${ }^{x}$ Ana Núñez Ares ${ }^{y}$ \\ Lorena López Roldán ${ }^{z}$ Carmen Abellán Martínez ${ }^{m}$ Abel Jesús Martínez Garcíay \\ Francisco Javier Michel de la Rosa ${ }^{A}$ Raúl Godoy Mayoral ${ }^{\mathrm{B}} \quad$ Miguel Ángel Martínez-García $^{\mathrm{d}}$ \\ ${ }^{a}$ Pneumology Service, Hospital Plató, Barcelona, Spain; ${ }^{b}$ Pneumology Service, Hospital Mutua de Terrassa, Terrassa,

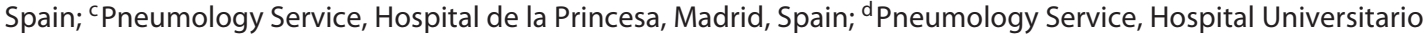 \\ y Politécnico de La Fe, Valencia, Spain; ${ }^{e}$ Pneumology Service, Instituto de Investigación Biomédica de Málaga

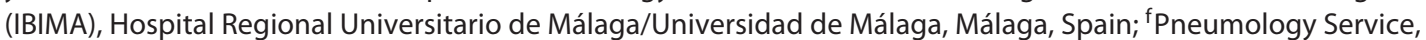 \\ Hospital Costa del Sol, Marbella, Spain; 9 Pneumology Service, Hospital Universitario La Paz-IS Carlos III, Madrid,

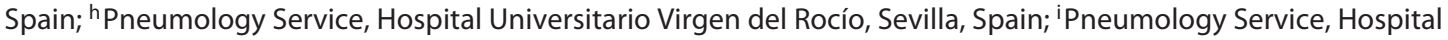 \\ de la Santa Creu i Sant Pau, Barcelona, Spain; 'Pneumology Service, Hospital de Viladecans, Viladecans, Spain; \\ kPneumology Service, Hospital Universitario Príncipe de Asturias, Alcalá de Henares, Spain; ' Respiratory Institute, \\ Hospital Clinic i Provincial, Barcelona, Spain; ${ }^{m}$ Pneumology Service, Hospital General Universitario Reina Sofía,

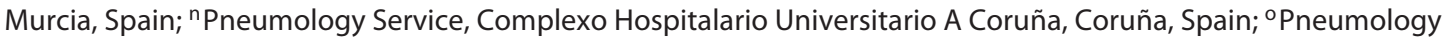 \\ Service, Hospital Universitario Central de Asturias, Oviedo, Spain; ${ }^{\mathrm{P} P n e u m o l o g y}$ Service, Hospital Universitario Lucus \\ Augustí, Lugo, Spain; ${ }^{9}$ Pneumology Service, Hospital de Cruces, Baracaldo, Spain; ${ }^{r}$ Pneumology Service, Hospital

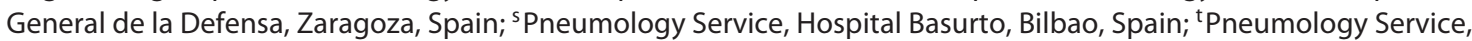 \\ Hospital Universitario Puerta de Hierro, Majadahonda, Spain; uPneumology Service, Hospital Universitario Infanta \\ Leonor, Madrid, Spain; ${ }^{\vee}$ Pneumology Service, Hospital Universitario Ramón y Cajal, Madrid, Spain; wPneumology \\ Service, Hospital Clínico San Carlos, Madrid, Spain; ${ }^{x}$ Pneumology Service, Hospital de Galdakao, Galdakao, Spain; \\ y Pneumology Service, Hospital General Universitario de Albacete, Albacete, Spain; ${ }^{2}$ Pneumology Service, Hospital \\ de Santa Marina, Bilbao, Spain; A Pneumology Service, Hospital Universitario Donostia, San Sebastian, Spain; \\ ${ }^{B}$ Pneumology Service, Hospital Universitario Nuestra Señora del Perpetuo Socorro, Albacete, Spain
}

\section{Keywords}

Antibiotic treatment $\cdot$ Bronchiectasis · Costs .

Hospitalization · Pseudomonas aeruginosa colonization

\begin{abstract}
Background: Knowing the cost of hospitalizations for exacerbation in bronchiectasis patients is essential to perform cost-effectiveness studies of treatments that aim to reduce exacerbations in these patients. Objectives: To find out the
\end{abstract}

\section{KARGER}

(c) 2018 S. Karger AG, Basel

E-Mail karger@karger.com

www.karger.com/res
David de la Rosa Carrillo

Pneumology Unit, Hospital Plató

Carrer Plató, 21

ES-08006 Barcelona (Spain)

E-Mail david.rosa23@gmail.com 
mean cost of hospitalizations due to exacerbations in bronchiectasis patients, and to identify factors associated with higher costs. Methods: Prospective, observational, multicenter study in adult bronchiectasis patients hospitalized due to exacerbation. All expenses from the patients' arrival at hospital to their discharge were calculated: diagnostic tests, treatments, transferals, home hospitalization, admission to convalescence centers, and hospitals' structural costs for each patient (each hospital's tariff for emergencies and 70\% of the price of a bed for each day in a hospital ward). Results: A total of 222 patients ( $52.7 \%$ men, mean age 71.8 years) admitted to 29 hospitals were included. Adding together all the expenses, the mean cost of the hospitalization was EUR $5,284.7$, most of which correspond to the hospital ward (86.9\%), and particularly to the hospitals' structural costs. The adjusted multivariate analysis showed that chronic bronchial infection by Pseudomonas aeruginosa, days spent in the hospital, and completing the treatment with home hospitalization were factors independently associated with a higher overall cost of the hospitalization. Conclusions: The mean cost of a hospitalization due to bronchiectasis exacerbation obtained from the individual data of each episode is higher than the cost per process calculated by the health authorities. The most determining factor of a higher cost is chronic bronchial infection due to $P$. aeruginosa, which leads to a longer hospital stay and the use of home hospitalization.

(c) 2018 S. Karger AG, Basel

\section{Introduction}

Non-cystic fibrosis bronchiectasis (hereafter BE) is a chronic airway disease concomitant with infectious exacerbations, even in its early stages. In more advanced stages, patients can suffer from chronic bronchial infection (CBI) due to potentially pathogenic microorganisms, which is associated with more symptoms, accelerated decline in lung function, quality of life deterioration, and increased exacerbations [1-3].

Recent decades have seen a progressive increase in the prevalence of BE, as well as resulting hospitalizations [47]. A history of frequent exacerbations and hospital admissions predict future mortality, hospital admissions, and exacerbations, and are associated with higher healthcare costs [8-10]. Furthermore, various studies have shown that $\mathrm{BE}$ is associated with high healthcare costs that increase with the severity of the disease, mainly due to hospitalizations and various treatments in the stable phase [10-13]. An analysis of patient databases in the United States estimated the cost of a hospitalization as
USD 7,827 [13], but no study has yet evaluated hospitalization costs on the basis of data obtained directly from the individual episodes of each patient.

Many of the treatments used in BE, such as inhaled antibiotics in more severe patients with CBI, aim to reduce the number of exacerbations, and could therefore contribute to the decrease in the number of hospitalizations. Given the high cost of such drugs, cost-efficiency or cost-utility studies are needed for an accurate assessment of their clinical benefit. However, these would require information that is as yet unavailable, such as the mean cost of hospitalizations due to BE exacerbations.

We therefore set about performing a prospective, multicenter study to establish the mean cost of a usual hospitalization due to a respiratory exacerbation in BE patients in Spain and identify the factors associated with higher costs.

\section{Material and Methods}

\section{Study Design}

This was a prospective, observational, and multicenter study involving hospitals from all over Spain. Data were used in accordance with the Data Protection Law 15/1999, authorization was given by each participating hospital's Ethics Committee for clinical research, and informed consent was obtained from all patients included in the study.

Adult patients diagnosed with BE via thoracic high-resolution computerized tomography, with a diagnosis of exacerbation of BE who satisfied the requirements of hospital admission [14], were included. With the intention of having a homogeneous sample, given the seasonal behavior of BE exacerbations, and to avoid any possible bias due to seasonality (as it would have been including the majority of patients in times of flu epidemic), no more than 2 patients were included per center in a single month.

Patients were excluded when they presented: cystic fibrosis; admission for other respiratory causes (pneumonia, pulmonary embolism); and traction BE. In order to have a patient population as homogeneous as possible, both in terms of the type of patient and the place of admission, we also excluded patients with a readmission less than 8 weeks after the previous discharge for a BE exacerbation, admission to an intensive care unit, and exacerbations treated directly with hospitalization at home $(\mathrm{HH})$ without actually entering hospital ward (although patients who completed their treatment with $\mathrm{HH}$ after admission to a ward were included).

We determined the sample size assuming an approximate prevalence in the general population of 100 cases per 100,000 inhabitants [5]. The minimum sample was calculated estimating a margin of error of 5\% and a confidence level of $85 \%$, establishing that 207 patients with complete data were required for the study.

\section{Variables}

The following data were collected: demographic data, clinical data (age at start of symptoms and diagnosis of BE, presence and habitual type of expectoration, scale of mMRC dyspnea, exacerba- 


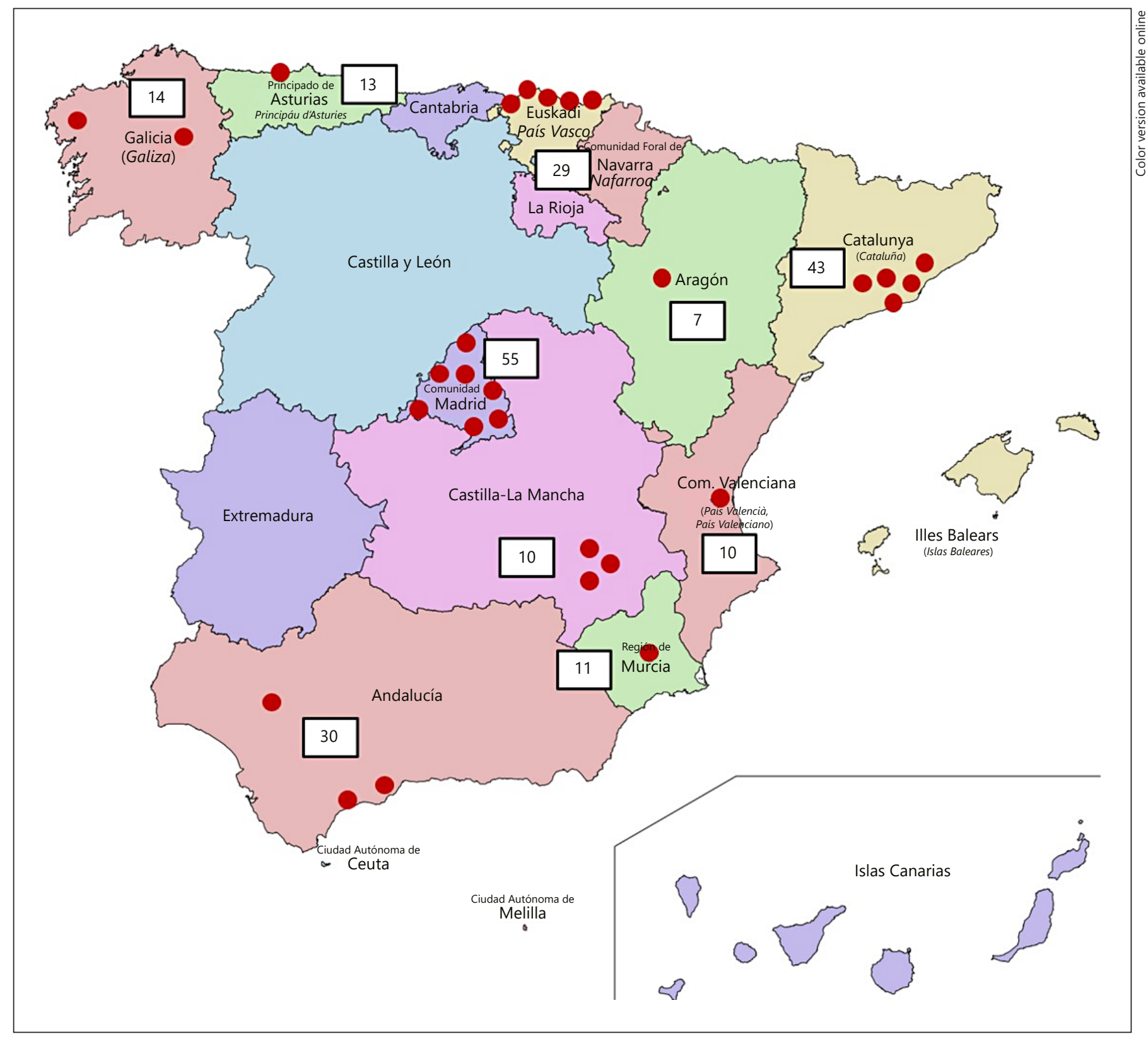

Fig. 1. Geographical distribution of centers participating in the study. Number of patients enrolled by autonomous community.

tions and admissions during the previous year, Charlson Comorbidity Index); BE etiology; etiological tests performed; radiological extension of BE; microbiological data; spirometric data; severity of $\mathrm{BE}$ according to Bronchiectasis Severity Index (BSI), FACED, and E-FACED scores $[8,15,16]$; time spent in the emergency department, in the hospital ward, and in the $\mathrm{HH}$. We also collected data on employment, salary, the need for sick leave of the patient during the exacerbation, and the need for sick leave of the caregiver.

All the expenses made from the patients' arrival at the emergency department to their final discharge (from the hospital or from the $\mathrm{HH}$ ) were collected: all complementary explorations undertaken; all treatments administered (including fungible materials, where applicable); any other expenses related or not directly related to the initial process; use of healthcare transportation; admissions into a convalescence center. Any pharmacological treatments, respiratory therapies (oxygen therapy, nebulizers), and pulmonary rehabilitation in the 2 months prior to admission and in the 2 months after discharge, were also collected. 
Assignation of Unit Costs

The company sales prices shown on the website of the General Council of Official Pharmacists' Associations, www.portalfarma.com (consulted on October 29, 2014) was used to calculate the cost of medication administered exclusively within the hospital setting. For the rest of drugs, the public sales prices presented in the Vademecum (year 2014) were used. The mean of the tariffs published in the Official Bulletins of the participating Autonomous Communities in 2014 were used for the cost of the $\mathrm{HH}$, complementary explorations, respiratory therapies, healthcare transportation, respiratory rehabilitation, and convalescence centers. Furthermore, the hospitals' structural costs for each patient were calculated and added to the total cost. We used each hospital's tariff for emergencies as well as the $70 \%$ of the price of a bed/ day in a hospital ward (corresponding to staff wages, electricity, water, gas, etc.), according the "Recommendations for the Homogenous Collection of Hospitalization Costs in the NHS" [17]. A complete list of the unit costs is available in online Supplementary File 1 (see www.karger.com/doi/10.1159/000489935 for all online suppl. material).

To calculate the indirect costs due to the loss of productivity of the patient and/or their caregiver, a cost of EUR 57.82 per day of sick leave was established, taking into account the average annual gross salary for the year 2014, according to the National Institute of Statistics. However, when decomposing the total cost of hospitalization into its different components, we only took into account the global direct costs.

\section{Statistical Analysis}

SPSS Statistics software, version 21.0 (SPSS, Chicago, IL, USA) was used for statistical analysis. Quantitative variables were tabulated as mean and standard deviation, and qualitative variables as absolute numbers and percentages. The Kolmogorov-Smirnov test was used to analyze the distribution of variables. The Mann-Whitney test was used to compare quantitative variables between two groups and the Kruskal-Wallis test for those between more than two groups. The $\chi^{2}$ test was used to compare qualitative variables. To evaluate differences in costs between subgroups of patients, we used the Student's $t$ test for independent groups.

A linear multiple regression was used for multivariate analyses, with variables chosen according to clinical criteria or via automatic selection methods. When a variable selection method was required, the backward method was used, whereby the variable from the model with the highest associated $p$ value and any value over 0.05 or 0.10 , as appropriate, were eliminated, based on the sum of type III squares.

\section{Results}

\section{Data prior to Hospital Admission}

Between October 2014 and September 2015, we included 222 patients $(52.7 \%$ men) with a mean age of 71.8 years, admitted to 29 hospitals $(44.6 \%$ secondary level hospitals, 55.4\% tertiary hospitals) from 10 different autonomous communities (Fig. 1). No patient was included twice. Table 1 presents the patients' general data. The

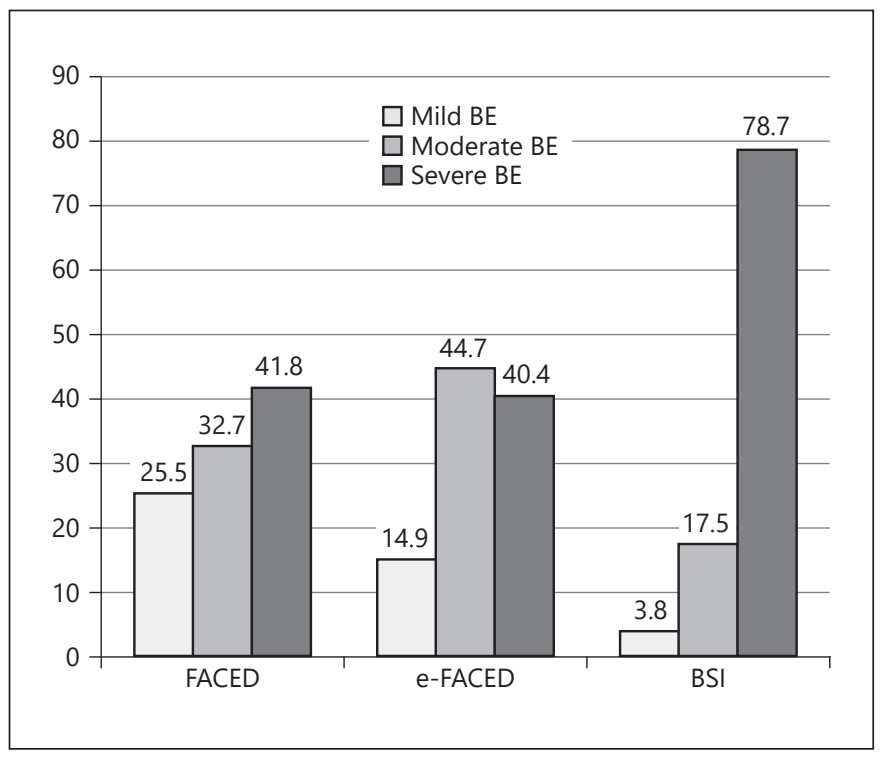

Fig. 2. Severity of bronchiectasis at the time of admission according to the various scales. BE, bronchiectasis; BSI, Bronchiectasis Severity Index.

most common $\mathrm{BE}$ etiologies were chronic obstructive pulmonary disease (COPD)-associated BE (24.3\%), postinfection (23.8\%), idiopathic (22.1\%), and posttuberculosis $(20.3 \%)$. In $62.6 \%$ of the cases, a complete blood analysis for etiological study had been carried out. Other etiological tests performed were: sweat test $(25.2 \%)$, serological response to vaccines $(10.1 \%)$, cystic fibrosis genetic test $(10.1 \%)$, and ciliary dyskinesia assessment (5.8\%). In some participating hospitals and in older patients, an etiological study was significantly less frequent (both $p<0.001$ ). $54.5 \%$ of the patients presented CBI by a potentially pathogenic microorganism, particularly Pseudomonas aeruginosa (PA) (80.4\%). According to the FACED and BSI indices, most of the patients had severe $\mathrm{BE}$, while according to the e-FACED the majority had moderate BE (Fig. 2). During the year prior to admission, $92.3 \%$ of the patients had presented exacerbations (mean $3.09 \pm 2.2$ ), and $66.2 \%$ had required hospitalization (mean $1.41 \pm 1.4)$.

\section{General Data on Hospital Admission}

The mean time spent in the emergency department was $14.1 \pm 15.1 \mathrm{~h}$, but $6.8 \%$ of patients were admitted on a scheduled basis, without being treated in the emergency room. The mean duration of the hospital stay (from arrival until discharge or transferal to $\mathrm{HH}$ ) was $10.8 \pm 5.5$ days. In $8.7 \%$ of cases, treatment was completed via $\mathrm{HH}$, 
Table 1. General baseline data of the included patients

\begin{tabular}{|c|c|}
\hline \multicolumn{2}{|l|}{ Demographic data } \\
\hline Men/women, \% & $52.7 / 47.3$ \\
\hline Age, years & $71.8 \pm 11.6(23-95)^{\mathrm{a}}$ \\
\hline Age at start of symptoms, years & $53.5 \pm 21.3(1-90)^{\mathrm{a}}$ \\
\hline Age at diagnosis, years & $61.6 \pm 16.9(8-90)^{\mathrm{a}}$ \\
\hline BMI & $26.8 \pm 5.0(15.8-46.4)^{\mathrm{a}}$ \\
\hline \multicolumn{2}{|l|}{ Smoking, \% } \\
\hline Yes & 4.5 \\
\hline No & 50 \\
\hline Ex-smoker & 45.5 \\
\hline Packs/year & $22.7 \pm 30.1(0-120)^{\mathrm{a}}$ \\
\hline \multicolumn{2}{|l|}{ Employment data, \% } \\
\hline Active employee & 7.7 \\
\hline Unemployed & 0.9 \\
\hline Retired & 68.9 \\
\hline Permanent disability & 6.3 \\
\hline Housewife & 15.8 \\
\hline Student & 0.5 \\
\hline \multicolumn{2}{|l|}{ Clinical data-symptoms, $\%$} \\
\hline \multicolumn{2}{|l|}{ Charlson Comorbidity Index } \\
\hline$\leq 3$ & 10 \\
\hline $4-6$ & 52.7 \\
\hline $7-9$ & 33.4 \\
\hline$>10$ & 4.3 \\
\hline \multicolumn{2}{|l|}{ Dyspnea } \\
\hline 0 & 7.7 \\
\hline 1 & 18.5 \\
\hline 2 & 34.2 \\
\hline 3 & 34.2 \\
\hline 4 & 5.4 \\
\hline Habitual expectoration & 85.1 \\
\hline \multicolumn{2}{|l|}{ Type of expectoration } \\
\hline White & 40.6 \\
\hline Mucopurulent & 44.8 \\
\hline Purulent & 13 \\
\hline Greyish & 1.6 \\
\hline \multicolumn{2}{|l|}{ Chronic bronchial infection } \\
\hline Active & 54.5 \\
\hline Prior & 12.2 \\
\hline
\end{tabular}

lasting a further $10.5 \pm 4.1$ days. These patients required less days in the hospitalization ward (7.58 vs. $11.1 ; p=$ $0.008)$ but more days of overall hospitalization period (18.1 vs. $11.1 ; p<0.001)$. Patients with CBI by PA required more days in the hospital ward (11.85 vs. 9.56 days; $p=0.002)$ and a longer overall hospitalization period (13.4 vs. 9.8; $p<0.001$ ). The parameters independently associated with a longer overall hospitalization period were the presence of CBI $(p=0.0327)$, particularly by PA $(p=0.002)$, two or more hospital admissions in the previous year $(p=0.041)$, and HH $(p=0.0079)$.

\begin{tabular}{lc} 
Respiratory function data & \\
$\mathrm{FVC}_{\text {. }} \%$ & $67.2 \pm 20.1(28-136)^{\mathrm{a}}$ \\
$\mathrm{FEV}_{1}, \%$ & $52.1 \pm 21.8(16-139)^{\mathrm{a}}$ \\
$\mathrm{FEV}_{1} / \mathrm{FVC}, \%$ & $57.8 \pm 14.1(30-98)^{\mathrm{a}}$ \\
\hline Microbiological data, \% of patients $^{\text {with }}$ CBI \\
Pseudomonas aeruginosa & 80.4 \\
Stenotrophomonas maltophilia & 5.3 \\
Haemophilus influenzae & 4.0 \\
Achromobacter xylosoxidans & 3.3 \\
Escherichia coli & 4.0 \\
Mycobacterium avium & 2.7 \\
Staphylococcus aureus & 2.7 \\
Klebsiella pneumoniae & 2.0 \\
Proteus mirabilis & 2.0 \\
Streptococcus pneumoniae & 2.0 \\
Aspergillus fumigatus & 1.3 \\
\hline Treatments, \% & \\
SABA & 22.5 \\
LABA & 88.7 \\
SAMA & 11.3 \\
LAMA & 65.3 \\
ICS & 86 \\
Systemic CCT & 14.9 \\
Mucolytic & 37.8 \\
Cyclical azithromycin & 29.7 \\
Nebulized ATB & 26.1 \\
Hypertonic saline solution & 6.8 \\
LTOT & 27.9 \\
\hline
\end{tabular}

ATB, antibiotic; BMI, body mass index; CCT, corticosteroids; $\mathrm{FEV}_{1}$, forced expiratory volume in $1 \mathrm{~s} ; \mathrm{FVC}$, forced vital capacity; ICS, inhaled corticosteroids; LABA, long-acting beta-agonist; LAMA, long-acting muscarinic antagonists; LTOT, long-term home oxygen therapy; SABA, short-acting beta-agonist; SAMA, short-acting muscarinic antagonists. ${ }^{a}$ Mean \pm SD (min-max).

\section{Cost of the Complementary Explorations and Treatments}

The mean cost of the complementary explorations undertaken in the emergency departments was EUR 147.1, and in the wards EUR 331.8 (Table 2). The online Supplementary File 2 presents details of these tests. These costs represented $35.4 \%$ of the emergency department expenses and $7.3 \%$ of hospital ward expenses (Fig. 3).

The mean cost of the treatments administered in the emergency departments was EUR 40.9, and in the hospital wards EUR 432.0 (Table 3). The online Supplementary File 3 presents details of the treatments administered. In both cases, the costs of intravenous antibiotics and inhaled treatments were particularly high, while there was also a significant outlay on non-respiratory treatments in 
Table 2. Cost of complementary explorations during stays in an emergency department or a hospital ward, EUR per patient (mean $\pm \mathrm{SD}$; min-max)

\begin{tabular}{lcc}
\hline & Emergency department & Hospital ward \\
\hline Venous access-probes & $11.7 \pm 7.9(0.06-53.2)$ & $8.6 \pm 10.2(0.06-52.8)$ \\
Image diagnosis & $26.9 \pm 36.8(0-240.3)$ & $84.8 \pm 131.4(0-1,192.5)$ \\
Laboratory tests & $75.9 \pm 51.6(0-307.9)$ & $154.3 \pm 158.8(0-983.6)$ \\
Microbiology & $31.3 \pm 32.6(0-142.4)$ & $59.9 \pm 55.5(0-234.9)$ \\
Bronchofibroscopy & - & $13.8 \pm 52.2(0-202.3)$ \\
Respiratory function tests & - & $10.4 \pm 34.6(0-202.3)$ \\
\hline Total cost & $147.1 \pm 86.8(0.6-652.5)$ & $331.8 \pm 299.5(0.06-1,819.6)$ \\
\hline
\end{tabular}

Table 3. Cost of the treatments administered during stays in an emergency department or hospital ward, EUR per patient (mean $\pm \mathrm{SD}$; min-max)

\begin{tabular}{lcc}
\hline & Emergency department & Hospital ward \\
\hline $\mathrm{O}_{2}$ & $0.51 \pm 0.45(0-3.8)$ & $0.53 \pm 0.4(0-1.9)$ \\
Systemic CCT & $1.2 \pm 1.45(0-10.6)$ & $8.4 \pm 8.9(0-48.4)$ \\
Inhalers (BD and ICS) & $11.1 \pm 23.3(0-128.2)$ & $58.5 \pm 46.6(0-265.3)$ \\
Theophylline & - & $0.07 \pm 0.4(0-3.24)$ \\
Oral ATB & $0.3 \pm 2.3(0-32.4)$ & $4.15 \pm 8.3(0-80.2)$ \\
Endovenous ATB & $16.7 \pm 23.6(0-198.6)$ & $234.2 \pm 209.7(0-1,315.4)$ \\
Nebulized ATB & $1.01 \pm 6.3(0-56.8)$ & $29.2 \pm 82.6(0-474.4)$ \\
Antifungicidals & - & $16.1 \pm 131.8(0-1,257.4)$ \\
Other antimicrobials & - & $12.6 \pm 140.3(0-2,051.5)$ \\
Other treatments & $6.9 \pm 14.7(0-94.2)$ & $57.8 \pm 91.4(0-956.3)$ \\
\hline Total cost & $40.9 \pm 58.0(0-585.4)$ & $432.0 \pm 389.8(16.13-2,828.2)$ \\
\hline
\end{tabular}

ATB, antibiotic; BD, bronchodilators; CCT, corticosteroids; ICS, inhaled corticosteroids; $\mathrm{O}_{2}$, oxygen therapy.

the wards. Treatments represented $9.1 \%$ of the total cost of emergency departments and $8.97 \%$ of that of hospital wards (Fig. 3). The cost of intravenous antibiotic therapy administered during the overall hospitalization period (emergency department, hospital ward, and $\mathrm{HH}$ ) was significantly higher in patients with CBI $(p=0.0011)$ and in patients who received inhaled antibiotics prior to admission $(p<0.0001)$.

\section{Global Costs}

The emergency department global cost, including the emergency department tariffs of each hospital, was EUR $432.1 \pm 342.4$. The cost of hospital stays in the wards, including transferals and structural costs for each center was EUR 4,665.9 $\pm 2,476.7$. In both cases, most of the costs corresponded to structural costs (Fig. 3). The mean cost of $\mathrm{HH}$ was EUR 2,575.5 $\pm 1,000.1$.

Cost of Hospitalizations due to

Bronchiectasis Exacerbation
Adding together all these costs (complementary explorations, treatments, $\mathrm{HH}$, transferals, emergency department tariffs, structural costs, and other expenses), the total mean cost of the overall hospitalization period was EUR 5,284.7 $\pm 2,445.7$. Only EUR 1,020.8 corresponded to the costs of complementary explorations, treatments, fungibles, and transferals, while the remainder corresponded to emergency department tariffs, hospital structural costs, and other expenses. Most of the global cost was attributed to hospital ward expenses (86.9\%) (Fig. 4). Patients who completed their treatment via $\mathrm{HH}$ incurred lower purchasing costs (EUR 803.6 vs. 1,038.9/patient; $p=0.033)$ but they tended to present a higher overall treatment cost (EUR 6,169.5 vs. 5,205.1/patient; $p=0.052$ ).

The univariate analysis showed a significantly higher cost in certain autonomous communities, in tertiary hospitals, in longer treatment periods, in patients with 


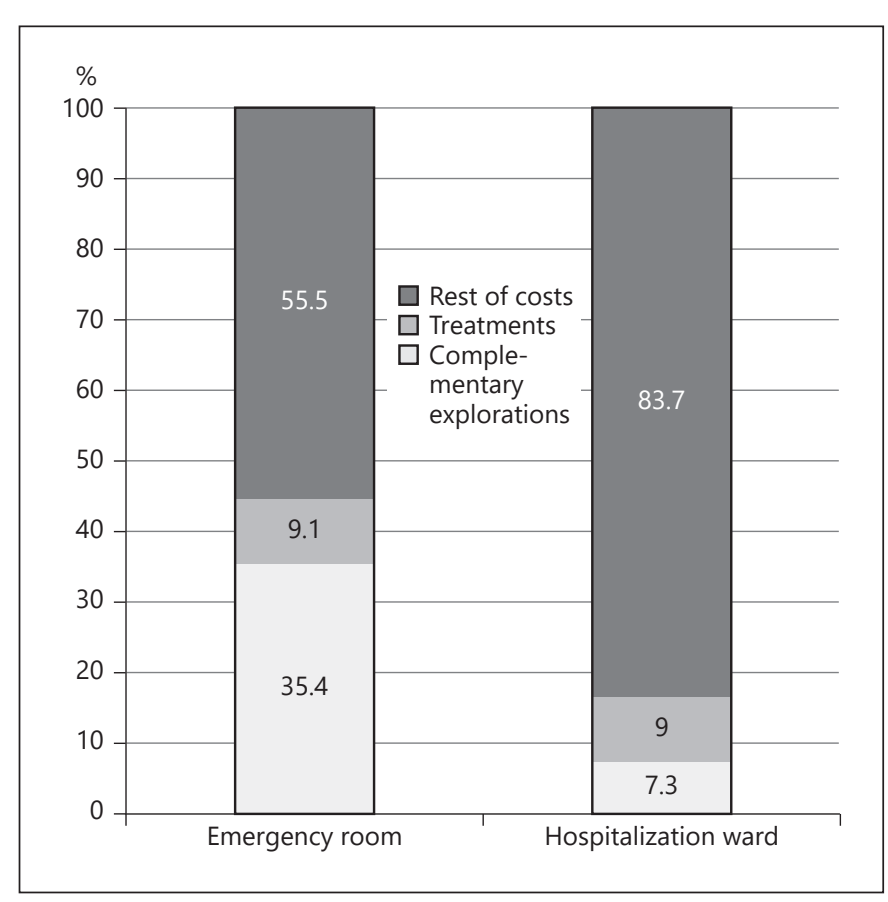

Fig. 3. Percentage of the cost of complementary explorations, treatments, and other expenses with respect to the cost of emergency departments and hospital wards.

CBI by PA or who were receiving inhaled antibiotic therapy prior to admission. We did not find any significant differences in the overall cost with respect to age, gender, etiology, severity of BE, Charlson Comorbidity Index, CBI by other microorganisms, and number of previous exacerbations or hospitalizations (see online additional data in suppl. File 4). The adjusted multivariate analysis showed that the variables independently associated with a higher overall cost were: $\mathrm{CBI}$ by PA, days spent in the hospital ward, and the completion of treatment via $\mathrm{HH}$ (see online additional data in suppl. File 5). Overall, these three parameters accounted for $90.7 \%$ of the variance.

\section{Indirect Costs}

Only $7.2 \%$ of the patients included required sick leave for an average of $13.44 \pm 9$ days (3-40), causing an additional indirect cost of EUR $776.9 \pm 520.6$ (EUR 173.5$2,312.8$ ). In $2.7 \%$ of the cases, it was the caregiver who required sick leave for $6.2 \pm 4.9$ days (2-16), with an indirect cost of EUR $356.5 \pm 286.6$ (EUR 115.6-925.1). Therefore, the overall cost of hospitalization, taking into account both direct and indirect expenses, was EUR $5,350.3 \pm 2,483.9$.

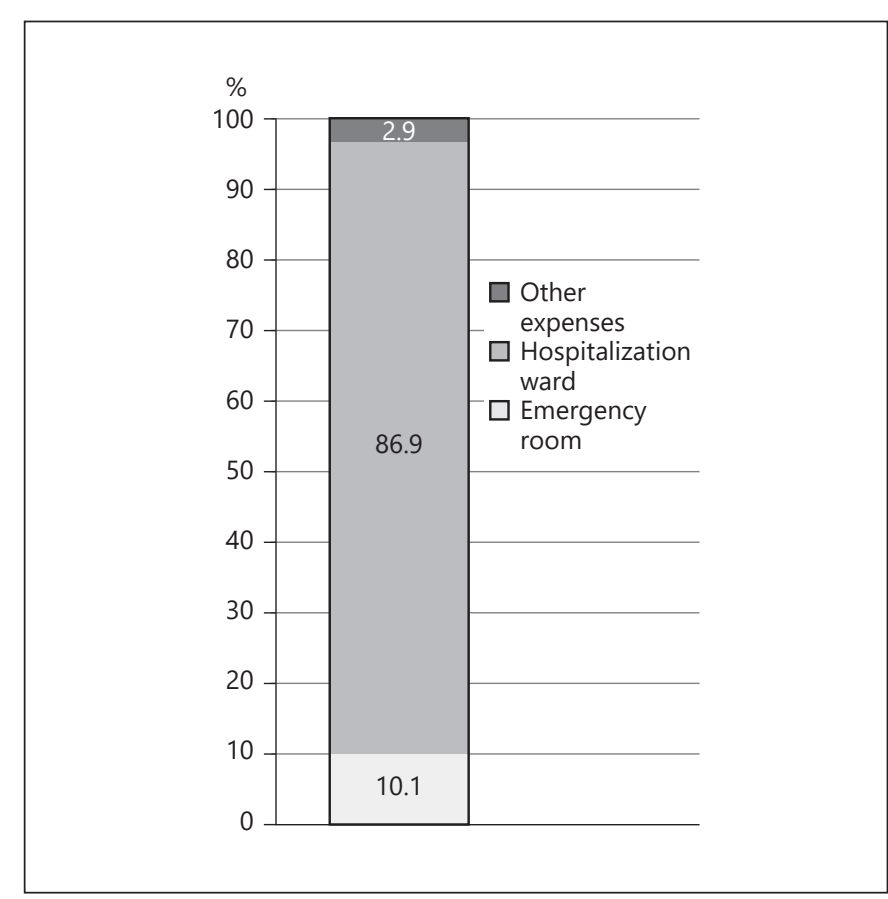

Fig. 4. Percentage of the cost of emergency departments and hospital wards with respect to the total cost.

\section{Data on the Post-Discharge Period}

After discharge, $15.1 \%$ of the patients required an increased dose of inhaled treatment, $21.5 \%$ required a different inhaled treatment, 9.6\% started inhaled antibiotic therapy, and $9.1 \%$ started long-term home oxygen therapy. Furthermore, $2.7 \%$ needed admission to a convalescence center, while $12.8 \%$ received respiratory rehabilitation. There was a significant increase in the treatment costs in the 2 months after discharge with respect to the 2 months prior to admission (Table 4). In the 2 months after discharge, $10.5 \%$ of the patients returned to an emergency department due to a new exacerbation, and 7.3\% needed readmission. The mortality rate during hospital stay and the 2 months after discharge was $1.4 \%$.

\section{Discussion}

This is the first study to directly evaluate the cost of hospitalization for BE exacerbation. It turned to be EUR 5,284.7, a figure that rises to EUR 5,350.3 if the indirect costs due to loss of productivity of the patient and/or their caregivers are taken into account. Most of the total expenditure corresponds to time spent in a hospital ward 
Table 4. Cost of treatment in the 2 months before admission and after discharge, EUR per patient (mean \pm SD)

\begin{tabular}{lccc}
\hline & Before admission & After discharge & $p$ value \\
\hline Inhalers (BD and ICS) & $183.8 \pm 81.3$ & $183.6 \pm 78.2$ & $\mathrm{~ns}$ \\
Systemic CCT $^{\mathrm{a}}$ & $0.8 \pm 2.4$ & $2.5 \pm 4.3$ & $<0.001$ \\
Other respiratory treatments & $12.8 \pm 33.4$ & $13.5 \pm 33.8$ & $\mathrm{~ns}$ \\
Mucolytics & $7.0 \pm 40.5$ & $4.2 \pm 6.4$ & $\mathrm{~ns}$ \\
Cyclical azithromycin & $10.1 \pm 17.2$ & $11.7 \pm 18.4$ & $\mathrm{~ns}$ \\
Oral ATB & $4.3 \pm 9.6$ & $14.5 \pm 88.5$ & $\mathrm{~ns}$ \\
Nebulized ATB & $275.0 \pm 506.6$ & $321.6 \pm 519.7$ & $\mathrm{~ns}$ \\
Hypertonic saline solution & $16.7 \pm 69.7$ & $14.6 \pm 69.3$ & $\mathrm{~ns}$ \\
Nebulizer & $13.4 \pm 31.3$ & $15.0 \pm 32.8$ & $\mathrm{~ns}$ \\
Oxygen therapy & $42.5 \pm 71.3$ & $54.2 \pm 76.2$ & 0.003 \\
Other treatments & $148.6 \pm 423.4$ & $142.9 \pm 421.0$ & 0.001 \\
\hline Total cost & $715.1 \pm 722.4$ & $778.6 \pm 718.9$ & 0.032 \\
\hline
\end{tabular}

ATB, antibiotic; BD, bronchodilators; CCT, corticosteroids; ICS, inhaled corticosteroids. ${ }^{\text {a }}$ Short courses of systemic steroids as part of the treatment of bronchial hyperresponsiveness associated with exacerbation.

(86.9\%), and more particularly to the hospital's structural maintenance (wages, electricity, water, maintenance, etc.). The parameters independently associated with higher costs were the presence of CBI by PA, the number of days spent in a ward, and the completion of treatment via $\mathrm{HH}$. This sum is markedly higher than the EUR 4,070.8 estimated by the Spanish Ministry of Health, according the relative weight of the diagnosis-related groups, for the year 2014.

The few studies that have analyzed the various aspects of the cost of $\mathrm{BE}[10-13]$ conclude that $\mathrm{BE}$ patients have a cost overrun with respect to patients without BE [11], right from the time of their diagnosis [12]. The annual cost of treatment is higher in patients with more severe BE, older age, more comorbidities, lower $\mathrm{FEV}_{1}$, more exacerbations or hospital admissions, CBI by PA, and an association with COPD. In more severe patients, most of the cost is due to exacerbations and inhaled antibiotic therapy [10]. Even fewer studies have evaluated the cost of hospital admission due to BE exacerbation. Seitz et al. [13] estimated the cost of an admission for BE exacerbation in the United States at USD 7,827. Most studies of this kind have analyzed big databases on patients from health insurance companies and calculated the direct healthcare costs related to data from patients with a diagnostic code for $\mathrm{BE}$, but this method fails to provide significant clinical data. Therefore, we decided to calculate the costs directly from the individual patient episodes, which allowed us to compare them with clinical information.

Some baseline data of our series are surprising, such as the high frequency of patients with COPD-associated BE, which probably implies the predominance of men, as opposed to other series with outpatients, where there is usually a majority of women. The COPD and BE association, and the possible causal relationship of the former with respect to latter, are questions that are currently being debated. Like in our case, other series have also found a high frequency of hospitalized patients who present both diseases synchronously $[6,7]$. In our study, the assignment of BE etiology depended on the criteria of the participant researcher, based on complementary tests and clinical history. Given the high age of the patients, both at the start of symptoms of BE (53.5 years), and at the time of diagnosis (61.6 years), it is understandable that in many cases, no exhaustive investigations were carried out to rule out etiologies that usually appear at younger ages [18]. Another striking fact is the high proportion of CBI by PA compared to other published series. One has to bear in mind that we only included patients who required hospitalization, whose previous history of exacerbations and hospitalizations was also greater than that of other series. $6.8 \%$ of them were hospitalized on a scheduled basis, specifically to administer intravenous antibiotics. Therefore, they were more severe patients, and it is logical that the percentage of CBI by PA has also been higher than those in other series [19-21].

We found a significant difference between the various autonomous communities with respect to the cost of hospitalization, because of the difference in the healthcare tariffs and in the severity of included patients. The overrun observed in tertiary hospitals is due to their higher price for a bed/day, and therefore higher structural costs. 
Despite that approximately $50 \%$ of the patients included had a Charlson Comorbidity Index score greater than 5 points, we found no relationship between this index and the total cost of hospitalization. At the time of carrying out this study, the Bronchiectasis Aetiology Comorbidity Index (BACI) [22] had not yet been published, so we do not have data in this regard. However, given that we did not find a relation between the cost of hospitalization, Charlson, and BSI, and taking into account the close correlation between the BACI and BSI, it is possible that the BACI would not have been significantly associated with the cost either. On the other hand, we also found no relationship between etiology and the cost of admission, despite the fact that some causes of $\mathrm{BE}$, such as immunodeficiencies, require expensive treatments. The small number of patients included with this type of disease (only 1 patient received treatment with intravenous immunoglobulins during hospitalization) influenced this lack of statistical relationship.

The cost was higher in patients with CBI by PA and in those receiving inhaled antibiotic treatment prior to hospitalization (all of these had CBI by PA). CBI by PA was also independently associated with a longer total treatment period. It is already well known that CBI by PA is associated with a poor clinical evolution in patients with $\mathrm{BE}$ [21], and so when these patients are admitted to hospital, they are treated with prolonged intravenous antibiotic therapy to eradicate or reduce this microorganism's bacterial load [23, 24], according to current guidelines [14]. The high percentage of CBI by PA in our study may have propitiated a longer hospitalization, which was even longer in those patients who completed their treatment with $\mathrm{HH}$. Most of the hospitalization expenses corresponded to hospital structural costs, which increase with every day spent in a ward. However, the expense was not reduced in those patients who were discharged early and continued treatment with $\mathrm{HH}$. More studies are needed to evaluate whether $\mathrm{HH}$ can be a strategy to reduce costs in patients in need of hospitalization for BE exacerbation, by comparing patients who receive treatment in a hospital ward with patients treated exclusively by $\mathrm{HH}$.

The data from our study allow us to deduce that the factor that most determines a prolonged hospital admission or the use of $\mathrm{HH}$, and therefore a higher cost of the treatment period, is CBI by PA. As this microorganism is associated with an increased rate of hospitalization [20] and a global increase in health costs from the moment of its first isolation [25], it is essential to find therapeutic strategies that reduce its clinical impact without increasing even further the overall healthcare expenses. A pro- longed administration of azithromycin reduces exacerbations in BE patients [26], but there is no evidence that it is useful in patients with CBI by PA. In these patients, inhaled antibiotics have been shown to reduce the number of exacerbations, hospitalizations, and hospital stay, although the few studies performed have included only a small number of patients [27]. The high cost of this treatment cries out for accurate cost-efficiency and cost-utility studies in larger series of patients. There is no evidence on other strategies to reduce $\mathrm{BE}$ hospitalizations or their cost, especially in patients with CBI by PA. Among the options that could be assessed, would be the direct $\mathrm{HH}$ (without previous stay in the hospital ward), the self-administration of intravenous antibiotic therapy at home or an intensive outpatient monitoring in day hospital [28].

We came up with a significantly higher hospitalization cost than the EUR 3,747-4,129 calculated for COPD patients by De Miguel-Díez et al. [29] in the period 20062010 in Spain. The mean hospitalization of 10.8 days in our series was also higher than that of COPD patients, which tended to be around 7 days $[29,30]$. With these data in mind, along with the increased prevalence of $\mathrm{BE}$ $[4,5]$, the higher incidence of hospitalizations for BE $[6$, $7,13]$, and the growing evidence of the latter's association with COPD [31-33], it is possible that the overall healthcare expenses due to $\mathrm{BE}$ are considerably higher than previously thought.

Finally, we evaluated the indirect costs due to the loss of productivity of the patient and/or their caregivers. However, when decomposing the total cost of hospitalization into its different components, we only took into account the direct costs, so that our results could be compared with those of other published series and those estimated by the Ministry of Health (which only included direct costs). Moreover, they proved to be of little relevance because most of the patients included were not occupationally active, and only a small proportion of the caregivers required taking time off work. We did not consider completing quality of life questionnaires during hospitalization, since we would not have had the same questionnaire carried out during clinical stability, before the hospitalization, in none of the included patients. Therefore, we could not have compared them and assess the possible negative impact of hospitalization on healthrelated quality of life.

Our study does have some limitations, such as the low number of patients included, which obliges us to be cautious about extrapolating the BE hospitalization costs. Nevertheless, the sample was very representative of Spain as it involved the participation of a large number of hos- 
pitals, from most of the country's autonomous communities. In order to obtain a homogeneous sample, despite the possible selection bias, we decided to exclude patients who had been hospitalized less than 8 weeks after a previous discharge, who had been admitted to an ICU or who were directly treated in $\mathrm{HH}$. Our aim was to assess the average cost of a usual hospitalization for $\mathrm{BE}$ in a ward, given that they account for the bulk of admissions for this disease. In addition, ICU patients and readmissions would have been confounding variables possibly related to a higher cost, and our intention was to assess which disease-specific variables were related to higher costs. The high proportion of patients with CBI by PA could also be considered a possible selection bias, but it should be borne in mind that only patients that required hospitalization were included, and so they logically presented more severe BE. Finally, the prices that we used correspond to Spain, which has a universal public health system, free for the patient. This means that our unit and overall costs are not directly applicable to other healthcare systems. However, we believe that the distribution of the hospitalization costs (emergency department, hospital ward, $\mathrm{HH}$, structural costs) as well as the factors associated with higher costs are likely to be similar in other countries, at least in those with a public health system.

In conclusion, the mean cost of a hospital admission due to exacerbation of BE in Spain, obtained by calculating the cost per patient per episode, is higher than the cost per process calculated by the health authorities. In the light of increased prevalence of $\mathrm{BE}$ and related hospitalizations, $\mathrm{BE}$ patients could be giving rise to considerable healthcare costs, thereby creating a need for population studies to analyze this phenomenon. In our study, the indirect costs due to loss of productivity had little relevance. Hospitals' structural costs are responsible for the bulk of hospitalization expenses, while the factors associated with higher costs are the presence of CBI by PA, the duration of the hospital stay, and the completion of treatment via $\mathrm{HH}$. As CBI by PA is also associated with a greater risk of hospitalization, specific treatments are also needed to reduce exacerbations and hospital admissions (particularly prolonged ones). Inhaled antibiotics seem to be the appropriate tool for reaching this objective, but their high price demands further clinical trials to evaluate their efficiency.

\section{Disclosure Statement}

All the authors of this paper declare that there are no conflicts of interest on the research.

\section{Funding Sources}

This work was supported by Praxis Pharmaceutical SL, VitoriaGasteiz, Spain, for the development of electronic databases, costs of statistical analysis, and translation of the manuscript. There was no intervention of the sponsor in the design of the study, collection and analysis of data, or elaboration of the manuscript.

\section{References}

1 Martínez-García MA, Soler-Cataluña JJ, Perpiñá-Tordera $\mathrm{M}$, Román-Sánchez $\mathrm{P}$, Soriano J: Factors associated with lung function decline in adult patients with stable non-cystic fibrosis bronchiectasis. Chest 2007; 132:15651572.

2 Martínez-García MA, Perpiñá Tordera M, Román Sánchez P, Soler Cataluña JJ: Internal consistency and validity of the Spanish version of the St. George's respiratory questionnaire for use in patients with clinically stable bronchiectasis. Arch Bronconeumol 2005;41: 110-117.

3 Olveira C, Olveira G, Espildora F, Giron RM, Muñoz G, Quittner AL, Martinez-Garcia MA: Validation of a Quality of Life Questionnaire for Bronchiectasis: psychometric analyses of the Spanish QOL-B-V3.0. Qual Life Res 2014; 23:1279-1292.

4 Quint JK, Millett ER, Joshi M, Navaratnam V, Thomas SL, Hurst JR, Smeeth L, Brown JS: Changes in the incidence, prevalence and mortality of bronchiectasis in the UK from 2004 to 2013: a population-based cohort study. Eur Respir J 2016;47:186-193.

5 Ringshausen FC, de Roux A, Diel R, Hohmann D, Welte T, Rademacher J: Bronchiectasis in Germany: a population-based estimation of disease prevalence. Eur Respir J 2015;46: 1805-1807

6 Ringshausen FC, de Roux A, Pletz MW, Hämäläinen N, Welte T, Rademacher J: Bronchiectasis-associated hospitalizations in Germany, 2005-2011: a population-based study of disease burden and trends. PLoS One 2013; 8:e71109.

7 Sánchez-Muñoz G, López de Andrés A, Jiménez-García R, Carrasco-Garrido P, Hernández-Barrera V, Pedraza-Serrano F, PuenteMaestu L, de Miguel-Díez J: Time trends in hospital admissions for bronchiectasis: analysis of the Spanish national hospital discharge data (2004 to 2013). PLoS One 2016;11: e0162282.
8 Chalmers JD, Goeminne P, Aliberti S, McDonnell MJ, Lonni S, Davidson J, Poppelwell L, Salih W, Pesci A, Dupont LJ, Fardon TC, De Soyza A, Hill AT: The Bronchiectasis Severity Index. Am J Respir Crit Care Med 2014; 189:576-585.

9 McDonnell MJ, Aliberti S, Goeminne PC, Dimakou K, Zucchetti SC, Davidson J, Ward C, Laffey JG, Finch S, Pesci A, Dupont LJ, Fardon TC, SkrbicD, Obradovic D, Cowman S, Loebinger MR, Rutherford RM, De Soyza A, Chalmers JD: Multidimensional severity assessment in bronchiectasis: an analysis of seven European cohorts. Thorax 2016;71:11101118.

10 de la Rosa D, Martínez-Garcia MA, Olveira C, Girón R, Máiz L, Prados C: Annual direct medical costs of bronchiectasis treatment: impact of severity, exacerbations, chronic bronchial colonization and chronic obstructive pulmonary disease coexistence. Chron Respir Dis, Epub ahead of print.
Cost of Hospitalizations due to

Bronchiectasis Exacerbation
Respiration 2018;96:406-416 DOI: $10.1159 / 000489935$ 
11 Weycker D, Edelsberg J, Oster G, Tino G: Prevalence and economic burden of bronchiectasis. Clin Pulm Med 2005;12:205-209.

12 Joish VN, Spilsbury-Cantalupo M, Operschall E, Luong Ba, Boklage S: Economic burden of non-cystic fibrosis bronchiectasis in the first year after diagnosis from a US health plan perspective. Appl Health Econ Health Policy 2013;11:299-304.

13 Seitz AE, Olivier KN, Steiner CA, Montes de Oca R, Holland SM, Prevots DR: Trends and burden of bronchiectasis-associated hospitalizations in the United States, 1993-2006. Chest 2010;138:944-949.

14 Martínez-García MA, Máiz L, Olveira C, Girón Moreno RM, de la Rosa Carrillo D, Blanco Aparicio M, Cantón Moreno R, Vendrell Relat M, Polverino E, de Gracia Roldán J, Prados Sánchez C: Spanish guidelines on treatment of bronchiectasis in adults (in Spanish). Arch Bronconeumol 2018;54:8898.

15 Martínez-García MA, de Gracia J, Vendrell Relat M, Girón R, Máiz Carro L, de la Rosa Carrillo D, Olveira C: Multidimensional approach to non-cystic fibrosis bronchiectasis. The FACED score. Eur Respir J 2014;43: 1357-1367.

16 Martinez-Garcia MA, Athanazio RA, Girón R, Máiz-Carro L, de la Rosa D, Olveira C, de Gracia J, Vendrell M, Prados-Sánchez C, Gramblicka G, Corso Pereira M, Lundgren FL, Fernandes De Figueiredo M, Arancibia F, Rached SZ: Predicting high risk of exacerbations in bronchiectasis: the E-FACED score. Int J Chron Obstruct Pulmon Dis 2017;12: 275-284.

17 Subdirección General de Información Sanitaria e Innovación: Guía de recomendaciones para la obtención homogénea de costes de hospitalización en el SNS. Ministerio de Sanidad, Servicios Sociales e Igualdad, 2013. http://www.msssi.gob.es/estadEstudios/estadisticas/inforRecopilaciones/anaDesarrolloGDR.htm (accessed December 6, 2017).
18 Buscot M, Pottier H, Marquette CH, Leroy S: Phenotyping adults with non-cystic fibrosis bronchiectasis: a 10 -year cohort study in a French regional university hospital center. Respiration 2016;92:1-8.

19 Angrill J, Agustí C, De Celis R, Rañó A, González J, Solé T, et al: Bacterial colonisation in patients with bronchiectasis: microbiological pattern and risk factors. Thorax 2002;57: 15-19.

20 McDonnell MJ, Jary HR, Perry A, MacFarlane JG, Hester KL, Small T, Molyneux C, Perry JD, Walton KE, De Soyza A: Non cystic fibrosis bronchiectasis: a longitudinal retrospective observational cohort study of Pseudomonas persistence and resistance. Respir Med 2015;109:716-726.

21 Finch S, McDonnell MJ, Abo-Leyah H, Aliberti S, Chalmers JD: A comprehensive analysis of the impact of Pseudomonas aeruginosa colonization on prognosis in adult bronchiectasis. Ann Am Thorac Soc 2015;12:1602-1611.

22 McDonnell MJ, Aliberti S, Goeminne PC, Restrepo MI, Finch S, Pesci A, Dupont LJ, Fardon TC, Wilson R, Loebinger MR, Skrbic D, Obradovic D, De Soyza A, Ward C, Laffey JG, Rutherford RM, Chalmers JD: Comorbidities and the risk of mortality in patients with bronchiectasis: an international cohort study. Lancet Respir Med 2016;4:969-979.

23 Orriols R, Hernando R, Ferrer A, Terradas S, Montoro B: Eradication therapy against $P$ seudomonas aeruginosa in non-cystic fibrosis bronchiectasis. Respiration 2015;90:299-305.

24 White L, Mirrani G, Grover M, Rollason J, Malin A, Suntharalingam J: Outcomes of Pseudomonas eradication therapy in patients with non-cystic fibrosis bronchiectasis. Respir Med 2012;106:356-360.

25 Blanchette CM, Noone JM, Stone G, Zacherle E, Patel RP, Howden R, Mapel D: Healthcare cost and utilization before and after diagnosis of Pseudomonas aeruginosa among patients with non-cystic fibrosis bronchiectasis in the US. Med Sci (Basel) DOI: 10.3390/medsci5040020.
26 Hill AT: Macrolides for clinically significant bronchiectasis in adults: who should receive this treatment? Chest 2016;150:1187-1193.

27 Yang JW, Fan LC, Lu HW, Miao XY, Mao B, $\mathrm{Xu} \mathrm{JF}$ : Efficacy and safety of long-term inhaled antibiotic for patients with non-cystic fibrosis bronchiectasis: a meta-analysis. Clin Respir J 2016;10:731-739.

28 Welsh EJ, Evans DJ, Fowler SJ, et al: Interventions for bronchiectasis: an overview of $\mathrm{Co}-$ chrane systematic reviews. Cochrane Database Syst Rev 2015;7:CD010337.

29 de Miguel-Díez J, Jiménez-García R, Hernández-Barrera V, Puente-Maestu L, RodríguezRodríguez P, López de Andrés A, CarrascoGarrido P: Trends in hospital admissions for acute exacerbation of COPD in Spain from 2006 to 2010. Respir Med 2013;107:717-723.

30 Harries TH, Thornton HV, Crichton S, Schofield P, Gilkes A, White PT: Length of stay of COPD hospital admissions between 2006 and 2010: a retrospective longitudinal study. Int J Chron Obstruct Pulmon Dis 2015;10:603611.

31 Gatheral T, Kumar N, Sansom B, Lai D, Nair A, Vlahos I, et al: COPD related bronchiectasis; independent impact on disease course and outcomes. COPD 2014;11:605-614.

$32 \mathrm{Du}$ Q, Jin J, Liu X, Sun AND: Bronchiectasis as a comorbidity of chronic obstructive pulmonary disease: a systematic review and meta-analysis. PLoS One 2016;11:e0150532.

33 De la Rosa D, Martínez-Garcia MA, Giron RM, Vendrell M, Olveira C, Borderias L, Maiz L, Torres A, Martínez-Moragon E, Rajas O, Casas F, Cordovilla R, de Gracia J: Clinical impact of chronic obstructive pulmonary disease on non-cystic fibrosis bronchiectasis. A study on 1,790 patients from the Spanish Bronchiectasis Historical Registry. PLoS One 2017;12:e0177931. 